\title{
Purposeful ensuring energy efficiency and environmental friendliness of waste and review of used local conservation laws
}

\author{
Gennady Korshunov ${ }^{1,2,{ }^{*}, \text { RemirSolnitsev }^{3} \text {, Sergey Polyakov }}{ }^{1}$, and Elena Frolova ${ }^{1}$ \\ ${ }^{1}$ IIQS dept. Saint-Petersburg State University of Airspace Instrumentation, 67, Bolshaya Morskaya St., Saint-Petersburg, 190000, Russian \\ Federation \\ ${ }^{2}$ CPSC high school, Peter the Great St. Petersburg Polytechnic University, 29, Polytechnicheskaya St.,Saint-Peterburg, 195251, Russian \\ Federation \\ ${ }^{3}$ CAD dept. Saint Petersburg Electrotechnical University "LETI" ul.ProfessoraPopova 5, 197376 St. Petersburg, Russian Federation
}

\begin{abstract}
The constant improvement of technologies is accompanied by an increase in requirements for the energy efficiency of industrial and residential facilities and the environmental friendliness of waste. The existing standards clearly formulate the concepts and limitations in this problem area. The developing class of cyber-physical systems accumulates the capabilities of cybernetics for modeling and controlling physical environments. Building on their basis not only monitoring systems, but also technology management is especially important in energy and ecology. When creating cyber-physical technology control systems, it is necessary to correctly formulate the goal. The article proposes the use of conservation laws in the format of their local forms. It shows how differential equations corresponding to these forms, transformed into an input-output form, are used to control the energy efficiency and environmental friendliness of waste and emissions from enterprises and other technogenic facilities.
\end{abstract}

\section{Introduction}

Science does not give a general definition of energy, the principle of its conservation means that there is something that remains constant [1]. Therefore, no matter what new ideas about the world will be led by future experiments, there remains something constant that can be called energy [2]. From a practical point of view, energy is defined as the ability of a given system of bodies under given conditions to perform a certain, quite definite amount of work. Energy, directly extracted in nature, which can be converted into electrical, thermal, mechanical, chemical, is called primary.

The active use of energy efficient technologies leads to a decrease in the negative impact on the environment. This is reflected in the increase in the environmental friendliness of waste resulting from man-made processes. The interaction of modern digital technologies and the physical environment includes monitoring the environment and managing its indicators based on modeling as part of cyber-physical systems (CPS) with feedback. In [3], it is proposed to create cyber-physical systems with a target orientation, to be considered within the framework of subject and problem areas. Depending on the problem area in which the CPS is created, mathematical models oriented to its features are applied. Most problems in physics lead to the need to solve differential equations. This can be explained by the fact that many physical laws are represented by differential equations, in relation to some functions, which characterize the processes of evolution of required values in space and time. The article suggests that mathematical models based on local forms of classical conservation laws should be used to achieve primary purposes in the problem areas of energy efficiency and waste ecology. Direct use of such models allows receive necessary quantitative estimations corresponding to tasks of measurements and monitoring. It has been shown that in order to solve the problems of control energy efficiency and environmental friendliness of waste, it is sufficient to convert differential equations to the form of "input-output".

\section{Materials and methods}

Conservation laws are fundamental physical laws, according to which, under certain conditions, some measurable physical quantities that characterize a closed physical system do not change over time. In general terms, the conservation law states: the change in time of a certain physical magnitude (PM) in an elementary volume is equal to the sum of the inflow-sink of this PM through its surface, taking into account the rate of generation or destruction of PM in this volume. The use of fundamental physical laws, which, first of all, include conservation laws (mass, energy, momentum) creates sufficient conditions for creating mathematical models of objects based on a theoretical analysis of the object under study and the physical processes occurring in it. These models, converted into an input-output form, are used to control energy efficiency and environmental

*Corresponding author: kgi@pantes.ru 
friendliness of waste and emissions from enterprises and other man-made facilities. This approach, based on the fundamental results of mathematical physics [4], makes it possible to describe the necessary physical processes with any available precision. The accuracy of representations and adequacy of models are conditioned by the level of knowledge achieved in this problem area and the formalization methods used. This corresponds to the concept cyber-physical systems [5..8], which represent the basis for innovative development. At the same time, the reduction of the mathematical models obtained to individual cases of application, as well as their transformation into the form of "input-output" have no common solution. Some examples of the application of the proposed approach are discussed below.

\subsection{Primary energy as a criterion for energy efficiency}

The energy conservation law equation can be represented as

$$
d(\rho E) / d t=-\operatorname{div}_{E}^{\mathrm{r}}+G_{E}
$$

where $E=e+\frac{v^{2}}{2}-$ totalenergyperunitmass, $e-$ internalenergyperunitmass, $v \quad$-speed, $\quad \rho E \quad-$ unitvolumeenergy, $J_{E}$ - energyfluxdensityvector, $G_{E}$ rateofgenerationorabsorptionofenergyperunitvolume.

Intheone-dimensionalcase,

theenergyfluxdensityismeasuredinJ $/ \mathrm{m}^{2} \mathrm{~s}$. An example of using this law is the heat conduction equation, which relates the change in temperature in time and space with the properties the medium

$$
d(\rho Q) / d t=-\operatorname{div}_{E}^{\mathrm{r}}+G_{Q}
$$

where $\mathrm{Q}$ is the amount of thermal energy per unit volume, $\mathrm{J} / \mathrm{m}^{3}$, is the vector of heat flux density, $\mathrm{J} / \mathrm{m}^{2} \mathrm{~s}$, $\mathrm{G}_{\mathrm{Q}}$ is the amount of thermal energy released per unit time in the considered elementary volume, $\mathrm{J} / \mathrm{m}^{3} \mathrm{~s}$.

In [9], energy efficiency is defined as a characteristic reflecting the ratio of the beneficial effect from the use of energy resources to the costs incurred in order to obtain such an effect, in relation to products, technological processes, and legal entities. It should be noted that the concept of energy efficiency is associated with the law of conservation of energy in all its transformations. In this sense, the concept of energy saving can be used only for one type of energy, which is "saved" by reducing the amount of energy in other forms.

Germany is a recognized global leader in energy and resource conservation with one of the most stringent laws and regulations in this area. Many European countries have adopted the experience of Germany, for example, the German EnEW standards. Most European countries use primary energy costs as a criterion for characterizing the energy efficiency of buildings.
Primary energy is determined by the amount of energy for the production of final energy, taken taking into account raw materials and processes of production, transformation and transmission of energy outside the enterprise. The description of thermal processes is possible on the basis of conservation laws and the equations of continuity arising from them. Physical laws in algebraic form are represented by equations of statics, and in differential form - by equations of dynamics. The laws reflect both the properties of individual elements of the object during the course of the selected processes, and express the conditions of balance and continuity of phase variables and describe the interactions between the elements of the system through the relations between the phase variables. The law of conservation of energy is a special case of the law of conservation, and its record in the form of an equation of heat (energy) balance is used for thermal calculations. The law of conservation of energy establishes that its change can be caused by movement inward or outward of the considered volume through the surface bounding it. In the balance equation, introduced energy is transferred to the left side, and expended energy to the right. In [10] the results of the joint work of European and Russian specialists in this area are presented.

\subsection{Models of monitoring and environmental friendliness of waste and emissions control}

In [11], environmental friendliness of waste is defined as the measurable and assessed properties of waste, representing its natural or deliberately provided ability, in all types of existence, not to have negative impacts within unacceptable limits on the environment, which is within a specified time in a certain proximity to the location of the waste. Conservation equations are used to determine the mass, density and concentration of waste and emissions. The mass conservation law has the form

$$
d \rho / d t=-\operatorname{divJ}_{\rho}^{\mathrm{r}}
$$

where $\rho$ is the mass density, ${ }^{I} \rho=\underset{\rho}{\mathrm{r}}$ is the mass flux density vector, $\stackrel{t}{v}$ is the mass transfer velocity vector.

In the one-dimensional case, when the velocity is directed along one axis of the Cartesian coordinate system, the mass flux density is measured in $\mathrm{kg} / \mathrm{m}^{2} \mathrm{~s}$.

The continuity equation in gas dynamics has the form:

$$
\frac{\partial \rho}{\partial t}+\rho \operatorname{div} V+V \operatorname{div} \rho=0
$$

where $\rho=\rho(x, y, z, t)$-fluid (orgas) flowdensity, $v=v(x, y, z, t)$ -thevelocityvectoroftheliquid (orgas) atthepointwithcoordinates $(x, y, z)$ atthemoment $t$.

Gas propagation models in the form of equations (4) have found application in analyzing the distribution of pollution emissions.

The main model of diffusion and convection is the turbulent diffusion equation: 


$$
\frac{\partial q}{\partial t}+\sum_{i=1}^{3} V_{i} \frac{\partial q}{\partial x_{i}}=\sum_{i=1}^{3} \frac{\partial}{\partial x_{i}} K_{i} \frac{\partial q}{\partial x_{i}}-\alpha q
$$

where $-q$ is the calculated mass (concentration) of pollutants; $x_{i^{-}}$coordinates; $V_{i}, K_{i^{-}}$components of the average velocity of impurity movement and exchange coefficient related to the direction of the $x_{i}$ axis $(i=1,2$, 3), $a$ - coefficient determining the change in concentration due to natural pollution compensation.

Various simplified formulas are used to determine the wind speed and diffusion coefficient, which vary depending on the state of the atmosphere.

For a single source of pollution, the equation of turbulent diffusion and convection (5) is presented in the form:

$$
\frac{\partial y}{\partial t}+V_{\xi} \frac{\partial y}{\partial \xi}+V_{\eta} \frac{\partial y}{\partial \eta}+V_{\zeta} \frac{\partial y}{\partial \zeta}=\frac{\partial}{\partial \xi} K_{\xi} \frac{\partial y}{\partial \xi}+\frac{\partial}{\partial \eta} K_{\eta} \frac{\partial y}{\partial \eta}+\frac{\partial}{\partial \zeta} K_{\zeta} \frac{\partial y}{\partial \zeta}+K_{1} \cdot X-K_{2} \cdot y
$$

where $Y(\xi, \eta, \zeta, t)$ - pollutantsconcentration, $V_{\xi} V_{\eta} V_{\zeta}$ theprojectionsofthepollutantstransfervelocityvectoronthe $\operatorname{axis} \xi \eta \zeta ; K_{\xi} K_{\eta} K_{\zeta}$ - diffusioncoefficientcomponents $K$.

Representation of physical processes using differential equations reflects physical laws. At the same time, for their practical use for monitoring and managing energy efficiency and environmental friendliness of emissions, they must be reduced to the "input-output" form [12].

In [13], it is shown how equation (6) is reduced to the "input-output" form (7):

$$
y(p, \xi, \eta, \zeta)\left(K_{2}+p\right)+K_{1} X(p)=e^{-\frac{K_{2}+p}{V_{\xi}}} \cdot\left(y_{0}\left(K_{2}+p\right)+X(p)\right)
$$

Emissionmonitoring control hasbeenreviewedinasufficientnumberofpublications. With regard to environmental management of waste and emissions, neutralization technologies and a number of other technologies presented in [14] are used. For example, emissions from enterprises using coal as a fuel contain fly ash with components, $\mathrm{CaO}$ and $\mathrm{MgO}$, which can partially neutralize $\mathrm{H}_{2} \mathrm{SO}_{4},-\mathrm{H}_{2} \mathrm{SO}_{4}+\mathrm{CaO}=\mathrm{CaSO}_{4}$ $+\mathrm{H}_{2} \mathrm{O}$.

$C O$ neutralization using $\mathrm{Pt}$ as a catalyst can be performed according to the formulas

$$
\begin{gathered}
\mathrm{O}_{2}+\mathrm{Pt} \stackrel{K_{1}^{ \pm}}{\longleftrightarrow} 2 \mathrm{PtO}, \\
\mathrm{CO}+\mathrm{Pt} \stackrel{K_{2}^{ \pm}}{\longleftrightarrow} \mathrm{PtCO}, \\
\mathrm{PtCO}+\mathrm{PtO} \stackrel{K_{3}}{\longleftrightarrow} 2 \mathrm{Pt}+\mathrm{CO}_{2},
\end{gathered}
$$

where: $\mathrm{O}_{2}$-molecularoxygen; $\mathrm{CO}$-carbonmonoxide; $\mathrm{CO}_{2}$-carbondioxide; $\mathrm{Pt}$-platinum (actingasacatalyst);

PtO, PtCO - intermediatesubstances; $K_{1}^{ \pm}, K_{2}^{ \pm}, K_{3}^{ \pm}-$ constants (atconstanttemperature) ofthereactionrate.
The process of neutralizing emissions by the $C O$ component is represented by a system of differential equations of the form [14]:

$$
\begin{aligned}
& \frac{d X_{O_{2}}}{d t}=-k_{1}^{\prime}-k_{1}^{\prime} X_{O_{2}} Q_{P t}^{2}+k_{-1}^{\prime} Q_{P t O}^{2}, \\
& \frac{d Q_{P_{t}}}{d t}=-k_{1}^{\prime \prime} X_{O_{2}} Q_{P_{t}}^{2}+k_{-1}^{\prime} Q_{P_{t} O}^{2}-k_{2} X_{C O} Q_{P_{t}}+k_{-2} X_{P_{t} C O}+k_{3}^{\prime} Q_{P_{t} C O} Q_{P_{t} O}, \\
& \frac{d Q_{P t O}}{d t}=k_{1}^{\prime \prime} X_{O_{2}} Q_{P_{t}}^{2}-k_{-1}^{\prime} Q_{P t O}^{2}-k_{3}^{\prime \prime} Q_{P t C O} Q_{P t O} \\
& \frac{d X_{C O}}{d t}=-k_{2} X_{C O} Q_{P t}+k_{-2} Q_{P t C O}, \\
& \frac{d Q_{P t C O}}{d t}=k_{2} X_{C O} Q_{P t}-k_{-2} Q_{P t C O}-k_{3}^{\prime \prime} Q_{P t C O} Q_{P t O}, \\
& \frac{d X_{\mathrm{CO}_{2}}}{d t}=k_{3}^{\prime \prime} Q_{P_{t} C O} Q_{P_{t O}}
\end{aligned}
$$

Where $\mathrm{X}_{\mathrm{O} 2}, \mathrm{X}_{\mathrm{CO}}, \mathrm{X}_{\mathrm{CO} 2}$-molarconcentration $\mathrm{O}_{2}, \mathrm{CO}$, $\mathrm{CO}_{2} ; Q_{P t}, Q_{P t O}, Q_{P t C O},-$ surfaceconcentrationPt, PtO, PtCO; $\quad k_{1}^{\prime} ; \quad k_{-1}^{\prime}{ }^{-} \quad$ secondorderforwardreactionratecoefficientsforthesurfacemediu $\mathrm{m}$;

$$
k_{1}^{\prime \prime}-
$$

second-

orderforwardreactionratecoefficientsforagasmedium; $k_{2}-$ secondorderforwardreactionratecoefficientsforagasmedium; $k_{-2}$ - firstorderfeedbackratecoefficients; $k_{3}^{\prime}$ - thirdorderforwardreactionratecoefficientsforthesurfacemediu $\mathrm{m}$; $k_{3}^{\prime \prime}-$ second-

orderforwardreactionratecoefficientsforthesurfacemediu $\mathrm{m}$. The solution to the system is given in [14]. Based on the simulation, the dynamics of changes in the concentrations of $\mathrm{CO}, \mathrm{CO}_{2}$ and other components of emissions at a fixed temperature and with a change in temperature was analyzed. It was revealed not only an increase in the efficiency of the catalyst with an increase in temperature, but also the required temperature regime for the release of emission concentrations into a given standard corridor in a minimum time. An important result is a decrease in the consumption of the catalyst substance due to the acceleration of the catalysis process.

For the problems of protecting underground metal structures from corrosion, which can be represented as a harmful product affecting the ecology of the environment, the parameter is the potential distribution determined by solving the Poisson equation [15] 


$$
\nabla^{2} \phi=-\rho_{S} \cdot \sum_{t=1}^{N} I_{t} \cdot \delta\left(P-\Psi_{t}\right)
$$

where $P$ is the observation point on the surface of the pipeline, $\Psi_{t}$ is the point of location of the source with the number $t$, It is the controlled protective current of the source.

Under the condition of making the assumptions of the potential linearity and equipotentiality of the pipeline cross-section, the equation describing the longitudinal distribution of the metal potential takes the form of a linear inhomogeneous differential equation

$$
\frac{d^{2} \phi_{M}}{d x^{2}}=r(P) \cdot i(P)
$$

where $r(P)$ - linear longitudinal resistance of the pipeline at the point under consideration; $i(P)$ - current flowing into the medium through the cross-section of the pipeline.

The distribution of the displacement of the regulated potential on the surface of the pipeline system is determined from the found current distribution along the pipeline system, and the "input-output" equation will have the form [15]

$$
\eta_{p}\left(x_{j}\right)=R_{c}\left(x_{j}\right) \cdot \eta\left(x_{j}\right)
$$

which determine the protection of the pipeline system against corrosion.

\section{Results}

The above models and methods are aimed at implementing the task of ensuring energy efficiency and environmental friendliness of emissions from enterprises. The given examples of different objects characterize the general approach. It consists in constructing dynamic, in the general case, models of energy and environmental processes based on differential equations. The compliance of such models with conservation laws allows to determine correctly the criteria for the subsequent control of energy efficiency and environmental friendliness of enterprises and other facilities.

\section{Discussion}

The proposed approach is based on modeling processes in physical environments and transforming models into an "input-output" form to implement control. This approach, associated with minimization or elimination of the "human factor", was proposed earlier as part of the concept of "nature-technogenics" [8] and fully corresponds to the concept of CPS. Obviously, there is no single solution to problems for various enterprises and the technologies existing in them. The selection of the required differential equations and the filling of their elements with concrete content presents individual research tasks in the relevant problem areas. For this purpose, experience from research and relevant results sufficient for inclusion in mathematical models can be used. Soft calculations and fuzzy logic that are not given in the article can be used to solve uncertainties. The division of tasks into monitoring and management, which has been examined using examples of energy efficiency and the environmental friendliness of enterprise waste, should be supplemented by a transition to digital management while minimizing the "human factor". Such examples are typical of $\mathrm{x}$ enterprises, where the objective is formulated as production efficiency. In this case, a transition (decomposition) from global goals in the problematic areas of energy, environment, information and others to specific goals of productivity, competitiveness, energy saving and others is of interest. Of interest is the analysis of the relationship between energy efficiency and waste ecology, which requires special consideration.

\section{Conclusion}

The solution of the problems of ensuring energy efficiency and environmental friendliness of waste of enterprises is currently largely achieved for monitoring, modeling, analysis and other procedures in the context of making managerial decisions. A number of works concern the creation of a CPS for monitoring. The approach presented in the article is aimed at solving the problem of managing energy efficiency and environmental friendliness of waste. The well-known results, including those proposed by the authors, can serve as a basis for creating input-output models for closed CPS with feedback. This approach is aimed at the transition to smart technologies. The creation of such technologies based on mathematical models is in demand in all types of industries.

\section{References}

[1] R.P. Feynman, The Feynman lectures on physics Vol. 1 (Addison-Wesley Publ.Co.Ink, Reading, Massachusetts, Palo Alto, London, 1963)

[2] A. Poincare, Thermodynamics (Moscow, Institute of computer research, 2005)

[3] G.I. Korshunov, S.L. Polyakov, J. Phys.: Conf. Ser. 1515, 2, 022065 (2020)

[4] A.N. Tikhonov, A.A. Samarsky, Equations of mathematical physics (Moscow, Nauka, 1977)

[5] E.A. Lee, S.A. Seshia, Introduction to Embedded Systems, A Cyber-Physical Systems Approach, Second Edition (MIT Press, 2017)

[6] I. Ruchkin, Integration of Modeling Methods for Cyber-Physical Systems, PhD thesis, Institute for Software Research School of Computer Science, Carnegie Mellon University, Pittsburgh, CMU-ISR18-107 (2019) 
[7] A.L. Ronzhin, O.O. Basov, B.V. Sokolov, R.M. Yusupov, Izv. vuzov, Priborostroenie 59, 11, 897-905 (2016)

[8] K. Babris, O. Nikiforova, U. Sukovskis, Applied Computer Systems Riga Technical University, Riga, Latvia 24, 1, 1-8 (2019)

[9] GOST R 56828.16-2017 National standard of the Russian Federation, Best available technology. Energy saving, Methodology for planning energy efficiency indicators (indicators) (2017)

[10] G.I. Korshunov, Management of the renovation of residential buildings to improve their energy efficiency (St. Petersburg, GUAP, 2015)

[11]GOST 30772-2001 Resource saving, Waste management, Terms and Definitions (2001)

[12] R.I. Solnitsev, Izvestiya, SPbGETU «LETI» 7, 2332 (2009)

[13] R.I. Solnitsev, G.I. Korshunov, Control systems «nature-technogenics» (St. Petersburg, Politekhnika, 2013)

[14]R.I. Solnitsev, G.I. Korshunov, A.A. Petrushevskaya, A.V. Paranichev, Cyber-physical systems in environmental safety and geomonitoring of vehicles, SPbGETU «LETI» (2019)

[15] G.I. Korshunov, A.V. Polyakov, E.A. Frolova, 14 Dep. At VINITI, 787-V2005, St. Petersburg (2005) 